Case Report

\title{
Glomus Tympanicum
}

\author{
Surg Vice Admiral VK Singh, AVSM,VSM*, Surg Cdr S Badhwar+, Surg Cdr J D'Souza\#, \\ Surg Cdr IK Indrajit**
}

MJAFI 2004; 60 : 200-203

Key Words : Glasscock Jackson Type 4; Glomus tympanicum; Paraganglioma

\section{Introduction}

$\mathrm{G}$ lomus tympanicum is the most common primary neoplasm of the middle ear and the second most common tumour of the temporal bone [1]. Although histologically benign, glomus tympanicum is slow growing, locally destructive, spreading along paths of least resistance. The most common presenting symptoms are conductive hearing loss and pulsatile tinnitus. CT and MRI scans are the primary imaging modalities used in evaluating the size and extent of glomus tumours. Superselective angiography with embolisation aids in identifying and blocking the feeding arteries, thereby reducing bleeding during surgery. Surgery and radiotherapy are the two modalities of treatment available. In this case report, we highlight the imaging and operative findings in glomus tympanicum, presenting in a 50 year lady.

\section{Case Report}

A 50 year old female presented to the otolaryngology OPD with a history of pulsatile tinnitus of 6 months duration and progressive, diminished, right-sided hearing of 3 months duration. She gave a history of intermittent otorrhoea from the right ear since 1992. A CT scan done at that time revealed findings suggestive of cholesteatoma. An exploratory tympanotomy done in right ear revealed a vascular mass and due to profuse bleeding the procedure was abandoned with a decision taken to evaluate further. The patient thereafter went outstation and was advised close follow up. On regular follow up, she experienced recurrence of ear discharge since Jun 2001, and presented to this hospital with a history of intermittent discharge and diminished hearing acuity in right ear. There was no history of vertigo. She was a known hypertensive since three years. There was no history of diabetes mellitus or ischaemic heart disease (IHD). Clinical examination revealed stenosis of the right external auditory canal and presence of a pulsatile mass behind the right tympanic membrane. Brown's sign was performed and was positive. There was no evidence of seventh or other cranial nerve deficit or vestibular finding. The systemic examination was essentially normal. With a diagnosis of pulsatile vascular mass of the right middle ear, she was subjected to further evaluation by imaging CT examination of the temporal bones performed on 27 Aug 2001 which demonstrated an enhancing mass occupying the middle ear, with extension to mastoid, infiltrating tegmen plate (Fig 1). The ossicles were surrounded by the mass, which engulfed the tympanic membrane. A MRI brain done on 29 Aug 2001 revealed evidence of an enhancing mass lesion (Fig 2), which was extending anteriorly into the eustachian tube, posteriorly into the mastoid cavity and superiorly in contact with the dura and was in close proximity to the jugular plate, jugular vein, sigmoid sinus, seventh nerve and labyrinth.

Superselective angiographic study performed on $10 \mathrm{Sep}$ 2001, showed a vascular tumour in the right middle ear supplied by right internal maxillary, posterior auricular, ascending pharyngeal arteries and tympanic branch of first part of maxillary artery (Fig 3A). These were selectively micro catheterised and embolised using Ivalon (Fig 3B). Based on the above clinicoimaging findings, a provisional diagnosis of glomus tympanicum (Glasscock Jackson Type 4) was made.

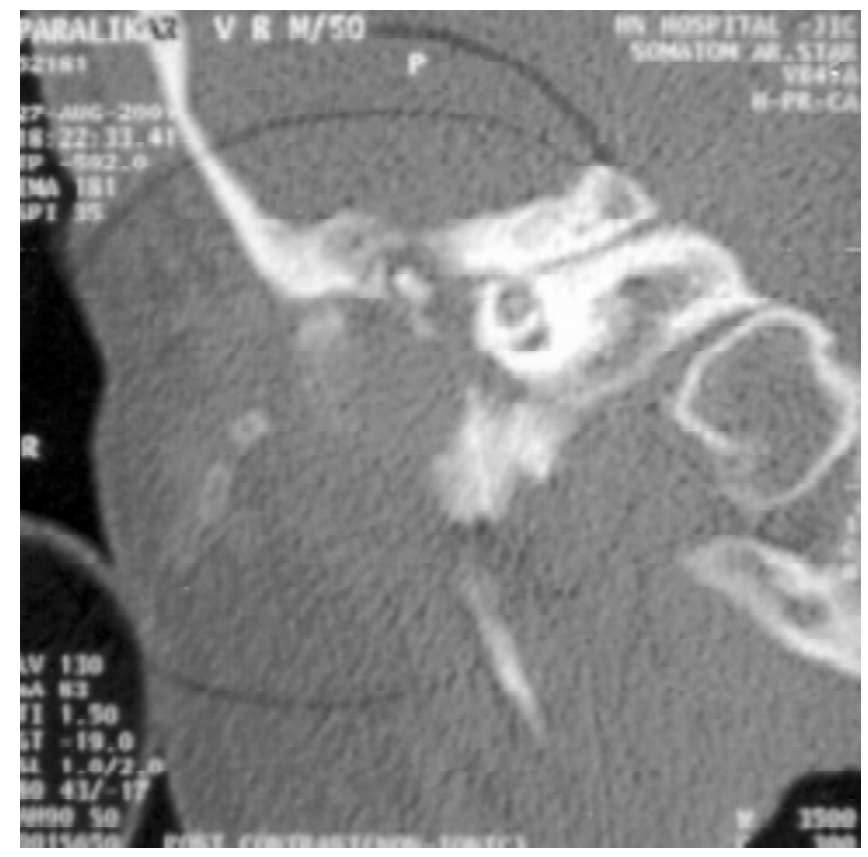

Fig. 1 : Coronal CT scan of the right temporal bone shows an infiltrating mass in the middle ear, extending to the mastoid, destroying the tegmen plate 


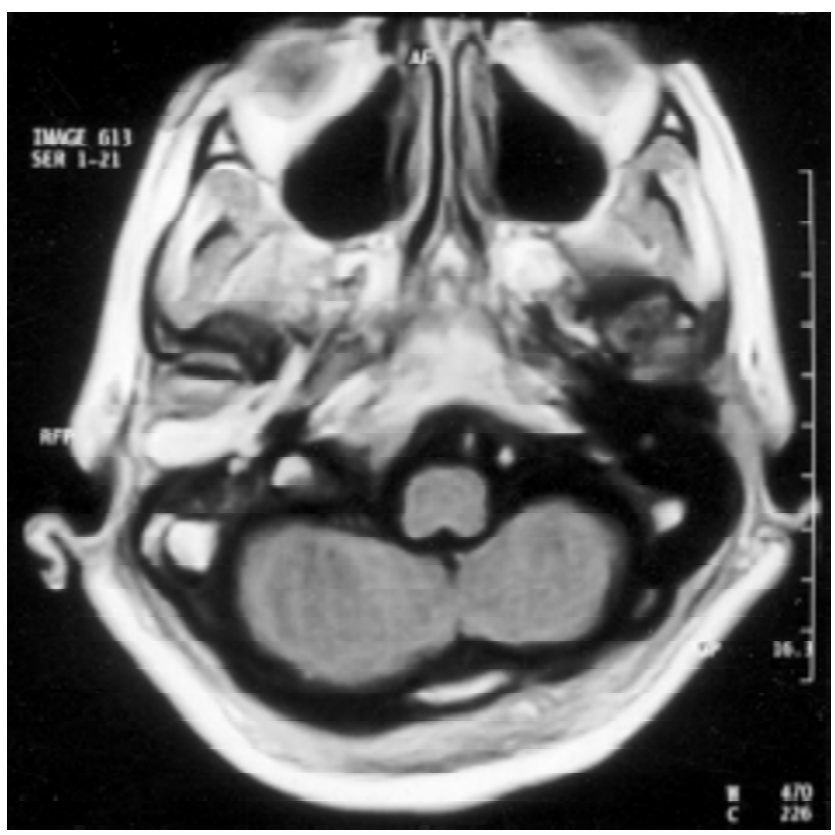

Fig. 2 : Post gladolinium axial MRI scans of the brain display an enhancing mass lesion, which extends anteriorly into the eustachean tube and laterally into the external auditory canal. The lesion is in close proximity to the jugular plate, jugular vein, sigmoid sinus, seventh nerve and the labyrinth

A tympanomastoid exploration was scheduled 24 hours after superselective angiography and embolisation.

On 11 Sep 2001, she underwent transmeatal, transmastoid excision of the glomus tumour by the post-aural route. Tumour was present in the external auditory meatus (Fig 4), filling the middle ear, extending towards the eustachian tube, the mastoid air cells, breaching superiorly the skull base at sinodural angle and tegmen and extending inferiorly into the hypotympanum (Fig 5). It was excised in toto, along with a wide meatoplasty with the resection status R0. The preoperative blood loss was $100 \mathrm{ml}$. In the postoperative period the cavity was clean and dry. A transient facial nerve weakness was present for a week, which subsided spontaneously. The lady was discharged on 25 Sep 2001 and is presently on regular follow up.

\section{Discussion}

Paragangliomas are benign, slow growing tumours that arise from neuroectodermal tissues. In the head and neck, two anatomic groups of paragangliomas can be differentiated : cervical paraganliomas and temporal bone (jugulotympanic) paragangliomas. The cervical group includes carotid body tumours and glomus vagale tumours, while the jugulotympanic comprises glomus jugulare and glomus tympanicum tumours. Glomus tympanicum tumours are more common than glomus tumours around the jugular vein, and are the most common primary neoplasms of the middle ear and the second most common tumour of the temporal bone [1].

In 1941, Guild first described "glomic tissue" in temporal bone as a vascular tissue in the dome of the

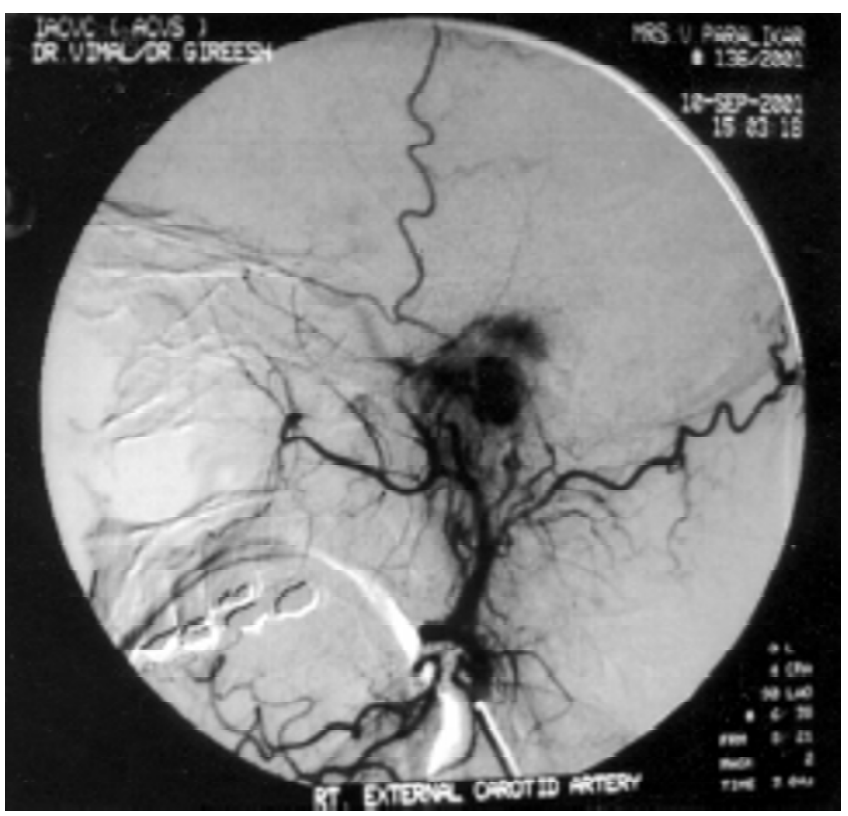

Fig. 3a : Superselective angiographic study of the right external carotid artery shows a hypervascular tumour mass in the right middle ear supplied by the internal maxillary artery, posterior auricular artery, ascending pharyngeal artery and tympanic branch of first part of maxillary artery

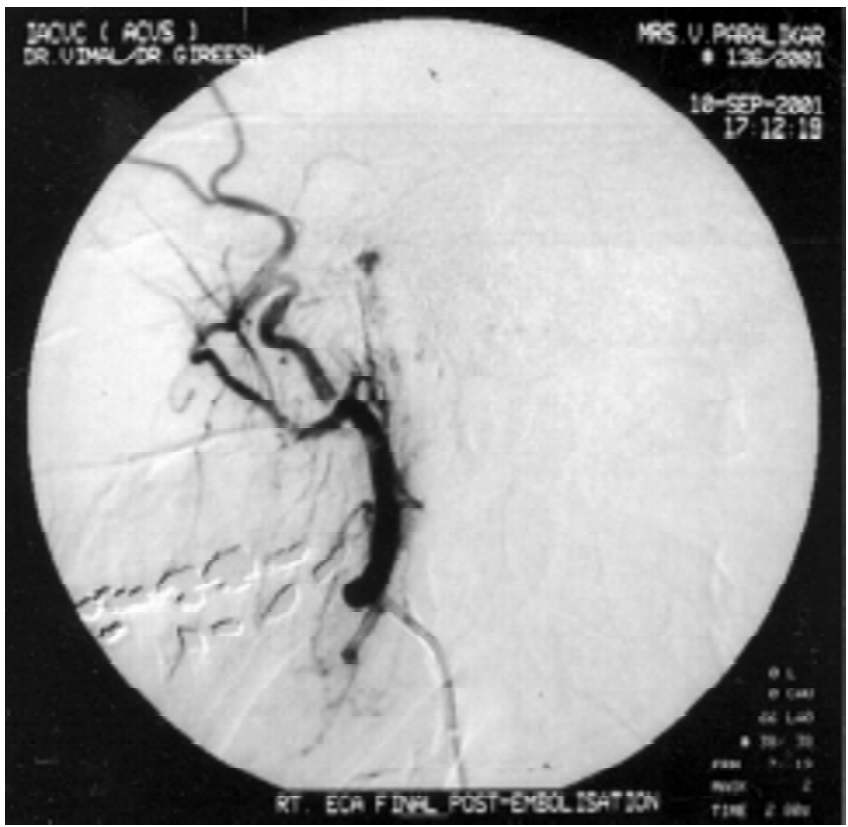

Fig. 3b : The post embolisation films show significant reduction of tumour vascularity

jugular bulb and the promontory of the middle ear [2]. Subsequently in 1945, Rosenwasser [3] reported a "carotid body tumour" of the middle ear and mastoid, referring to it as glomus tumour. Later, Guilford and Alford coined the term glomus tympanicum to describe those paragangliomas limited to the middle ear.

Paraganglia of the temporal bone are ovoid, lobulated bodies, usually found accompanying Jacobson's nerve (inferior tympanic branch of the glossopharyngeal nerve) 


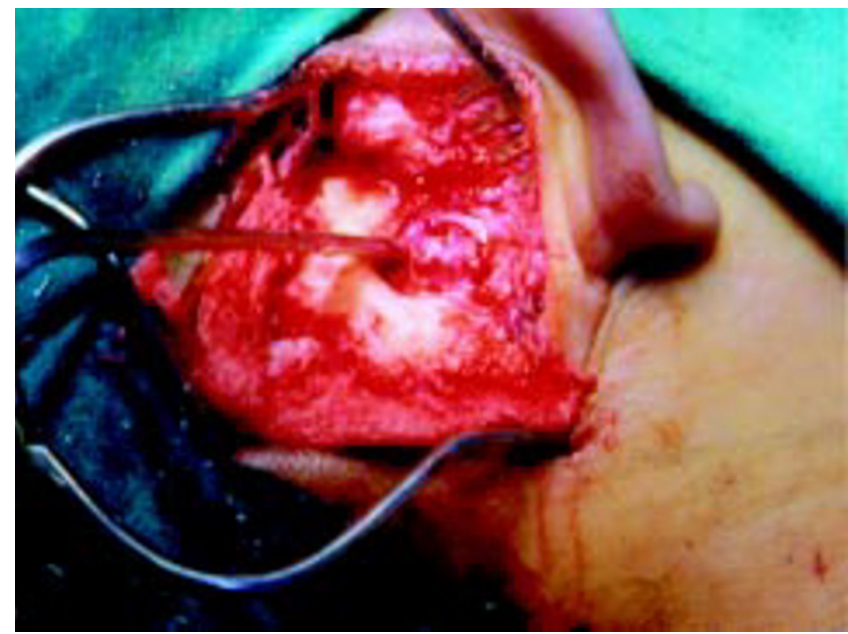

Fig. 4 : Pre-operative image showing a vascular mass in right external auditory meatus

or Arnold's nerve (mastoid branch of the vagus nerve) or in the adventitia of the jugular bulb. Serving as baroreceptors, paraganglia sense and regulate oxygen pressure in middle ear and mastoid. Microscopically, they consist of clusters of Type I or catecholamine containing chief cells ("zellballen") and Type II or sustentacular cells (modified Schwann cells), intimately interlaced with a rich network of capillaries and venules [4]. Catecholamine secreting or "functional" tumours occur in $1 \%-3 \%$ of cases. Multicentricity is found in about $5-15 \%$ of non-familial form patients. The incidence of malignancy in glomus tumours is believed to be low $(<5 \%)$.

Although histologically benign, glomus tumours are slow growing, locally destructive non-metastasizing, spreading along paths of least resistance. Spread is multidirectional and simultaneous. The main routes of spread are the air cell tracts of the temporal bone, but spread through and beyond the temporal bone is not uncommon, via eustachian tube, vascular lumens, and neurovascular foramina.

Patient age averages 50 to 60 years at presentation. The female : male incidence ratio is $4: 1$. The most common presenting symptoms include conductive hearing loss and pulsatile tinnitus [1]. Conductive hearing loss occurs when tumour impairs the normal vibration of the ossicles or bones behind the eardrum. A sensorineural hearing loss and /or dizziness can result rarely, if the tumour has invaded the inner ear. Other symptoms may include aural haemorrhage or otorrhea, otalgia and facial palsy. Aquino's sign is the blanching of the tympanic mass with gentle pressure on the carotid artery, while Brown's sign describes the pulsation elicited by pneumatic compression that is abolished with further compression. On physical examination, the hallmark of a jugulotympanic glomus tumour is a reddish-blue mass

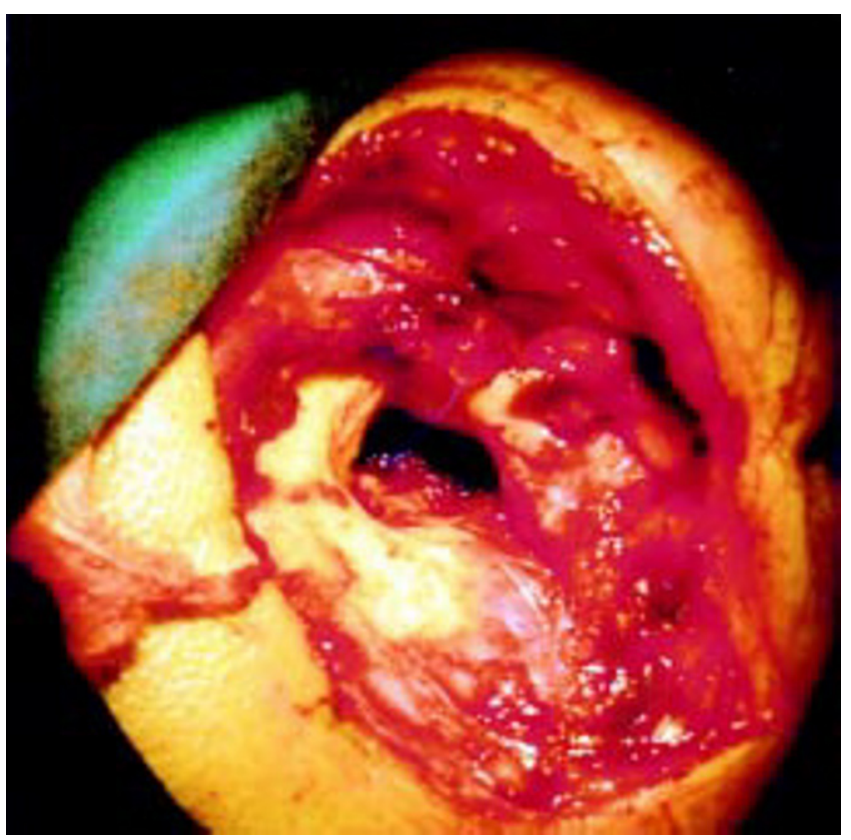

Fig. 5 : Per-operative image showing glomus tumour extending towards the right hypotympanum

seen behind the tympanic membrane. Hypertension, tachycardia, tremor, or complaints of vascular headaches alerts the possibility of a functional tumour.

Currently popular classification of glomus tympanicum tumour includes 'Fisch and Oldering' and 'Glasscock and Jackson'. The Glasscock and Jackson's system [5] classifies glomus tympanicum by area and degree of involvement into four types. While Type 1 glomus tumours are limited to the promontory, Type 2 denotes tumour completely filling the middle ear. Type 3 indicates tumour extending further into mastoid, whereas Type 4 glomus tumours spread into external auditory canal and may have intracranial extension.

On CT scan, glomus tympanicum appears as a soft tissue mass abutting the promontory of the middle ear [6]. There may be displacement of ossicles or bony erosion of the tympanic cavity. The finding of air or bone between the tumour and the jugular bulb virtually assures the diagnosis of a tympanicum. CT scans are best for evaluating bony destruction and erosion, which is a hallmark of jugulotympanic glomus tumours. MRI is usually better than CT for delineating tumour edges and intracranial extent. It is also better for evaluating the relationship of the tumour to adjacent jugular vein, carotid artery, membranous labyrinth and cranial nerves [7].

Angiography recognizes the primary feeding blood supply to the lesion, helps in detecting multicentric tumours, identifies intrasinus and intravenous extension, provides further information on flow in contralateral sigmoid and internal jugular vein and allows for possible pre-operative embolisation. Pre-operative embolization 
of feeding vessels is useful, if performed within 48 hours of planned surgery thereby greatly reducing the blood supply to the tumour, diminishing the need for transfusion, allowing the procedure to be performed with greater safety.

The treatment of glomus tympanicum, largely takes into account the patient's age, the site, size, and extent of the tumour, the rate of symptom progression, the preoperative cranial nerve status, the possibility of multicentricity and neurosecretory status and lastly the patient preference. Surgery and radiotherapy are the two modalities of treatment available [8]. In general, healthy younger patients or patients with large tumours with pre-existing ipsilateral cranial nerve deficits or patients with secreting tumours require surgical resection, while elderly patients with poor pulmonary function or other complicating medical conditions should consider radiotherapy. Unlike carotid paragangliomas, jugulotympanic paraganglioma are considered radiosensitive and the goal of radiotherapy is arrest of tumour growth. The risks of radiotherapy include tumour regrowth, late-onset cranial nerve defects and osteoradionecrosis of temporal bone. The surgical goal is total or near-total removal. Surgical risks include cranial nerve defects, vascular injury and bleeding and cerebrospinal fluid leak.

The size and the extent of the glomus tumour determine the surgical procedure needed [8]. Type 1 glomus tympanicum tumours are generally approached by trascanal tympanotomy. The Type 3 tumours require mastoidectomy with extended facial recess approach. Type 4 glomus tumours need intracranial surgical excision of the tumour, with assistance from a neurosurgeon. Patients with multicentric tumours require careful consideration, as bilateral lower cranial nerve deficits can be distressing. Residual tumour may require postoperative radiotherapy or observation with serial MRIs with long term follow up with serial otoscopic examinations.

\section{References}

1. O'Leary MJ, Shelton C, Giddings NA, Kwartler J, Brackmann DE. Glomus tympanicum tumours : a clinical perspective. Laryngoscope 1991;101:1038-43.

2. Karas DE, Kwartler JA. Glomus tumours : a fifty year historical perspective. Am J Otol 1993;14:495-500.

3. Stewart KL. Paragangliomas of the temporal bone. Am J Otolaryngol 1993;14:219-26.

4. Manolidis S, Shohet JA, Jackson CG, Glasscock ME. Malignant glomus tumours. Laryngoscope 1999;109:30-4.

5. Jackson CG, Glasscock ME, Harris PF. Glomus tumours : Diagnosis, classification and management of large lesions. Otolaryngol 1982;08:401-6.

6. Noujaim SE, Pattekar MA. Paraganglioma of the temporal bone : role of magnetic resonance imaging versus computed tomography. Top Magn Reson Imaging 2000;11:108-22.

7. Vogl TJ; Mack MG; Juergens M, Bergman C et al. Skull base tumours gadodiamide injection-enhanced MR imaging. Radiology 1993;188:339-46.

8. Sen C, Hague K, Kacchara R. Jugular foramen : microscopic anatomic features and implications for neural preservation with reference to glomus tumours involving the temporal bone. Neurosurgery 2001;48:838-47.

\section{MEDICAL RECORDS GONE WRONG}

These are actual, unedited doctor's notes on patients charts :

- The patient is tearful and crying constantly; she also appears to be depressed.

- Discharge status; alive but without permission.

- Healthy appearing decrepit 69 years old male, mentally alert but forgetful.

- The patient refused an autopsy. 